\title{
Sequential CD19/22 CAR T-cell immunotherapy following autologous stem cell transplantation for central nervous system lymphoma
}

\author{
Jiaying Wu (iD ${ }^{1}$, Fankai Meng ${ }^{1}$, Yang Cao ${ }^{1}$, Yicheng Zhang ${ }^{1}$, Xiaojian Zhu ${ }^{1}{ }^{1}$, Na Wang ${ }^{1}$, Jue Wang ${ }^{1}$, Lifang Huang ${ }^{1}$, \\ Jianfeng Zhou (iD ${ }^{1 凶}$ and Yi Xiao iD ${ }^{1 凶}$
}

(c) The Author(s) 2021

\begin{abstract}
Chimeric antigen receptor (CAR) T-cell immunotherapy following autologous stem cell transplantation (ASCT) is a promising method for refractory or relapsed multiple myeloma, but explicit data for central nervous system lymphoma (CNSL) are lacking. Here, we treated 13 CNSL patients with ASCT sequential CD19/22 CAR T-cell infusion and simultaneously evaluated the clinical efficacy and toxicity. The 13 CNSL patients analyzed included four primary CNSL and nine secondary CNSL patients. Patients 1 and 10, who had complete remission status before enrollment, maintained clinical efficacy without recurrence. Nine of the remaining 11 patients responded to our protocol with a median durable time of 14.03 months, and the overall response and complete remission rate were $81.81 \%$ and $54.55 \%$, respectively. No patient suffered grades 3-4 cytokine-release syndrome (CRS), and only patient 10 experienced severe immune effector cell-associated neurotoxicity syndrome (ICANS). In addition, increases in serum ferritin and interleukin- 6 levels were often accompanied by CRS and ICANS. After a median follow-up time of 14.20 months, the estimated 1-year progressionfree survival and overall survival rates were $74.59 \%$ and $82.50 \%$, respectively. Sequential CD19/22 CAR T-cell immunotherapy following ASCT as a novel method for CNSL appears to have encouraging long-term efficacy with relatively manageable side effects.
\end{abstract}

Blood Cancer Journal (2021)11:131; https://doi.org/10.1038/s41408-021-00523-2

\section{INTRODUCTIONS}

Central nervous system lymphoma (CNSL) is a lethal disorder with a poor prognosis, and it includes primary and secondary subtypes. Primary CNSL (PCNSL) is a rare subtype of extranodal non-Hodgkin lymphoma $(\mathrm{NHL})$ that mainly involves the brain, eyes, leptomeninges and spinal cord without evidence of systemic NHL. Secondary CNSL (SCNSL) refers to systemic NHL with central nervous system (CNS) involvement or relapse [1, 2]. In recent years, aggressive high-dose methotrexate (HD-MTX)-based induction chemotherapy and autologous stem cell transplantation (ASCT) consolidation therapy have improved the clinical outcomes of CNSL $[3,4]$. However, nearly three-fifths of PCNSLs appear to have refractory/relapsed $(r / r)$ status, and fewer than $20 \%$ of patients with secondary CNS involvement or relapse of malignant lymphoma can achieve long-term survival [5-7]; therefore, more encouraging treatment strategies for CNSL need to be investigated.

Chimeric antigen receptor (CAR) T-cells serve as a novel immunotherapy method for $r / r$ hematopoietic malignancies that result in a favorable clinical prognosis [8-10]; The "cocktail" treatment can effectively prevent tumor recurrence due to antigen escape [11-13]. However, the application of CAR T-cell immunotherapy in CNSL has been restricted due to concerns about the possibility of related severe cytokine-release syndrome (CRS) and immune effector cell-associated neurotoxicity syndrome (ICANS) [14]. Tu et al. [15] reported satisfactory clinical prognosis and controllable adverse events in a $\mathrm{r} / \mathrm{r}$ primary CNS diffuse largecell lymphoma (DLBCL) patient who received CD19/70 CAR T-cell infusion, indicating that CNSL was no longer an absolute contraindication for CAR T-cell immunotherapy. Another study conducted by our department also showed an objective response in CNSL patients who received CD19/ 22 CAR T-cell infusion, but only one complete remission (CR) case enrolled in another clinical trial of ASCT sequential CD19/22 CAR T-cell infusion achieved long-term progression/relapse-free survival; this finding demonstrated that separate CAR T-cell immunotherapy was effective but not long-lasting for CNSL, and the new model of CAR T-cell immunotherapy following ASCT provided a promising direction and option for the treatment of CNSL [16].

Here, we reported our findings for a total of 13 CNSL patients who underwent sequential CD19/22 CAR T-cell immunotherapy following ASCT based on preliminary experience. Our results indicate that sequential CD19/22 CAR T-cell immunotherapy following ASCT is a novel and promising method for CNSL patients to achieve long-term remission with manageable side effects.

\section{MATERIALS AND METHODS \\ Study design}

The present study was based on single-center, open-label, single-arm clinical trial (ChiCTR-OPN-16009847) data from January 1, 2019, to February 


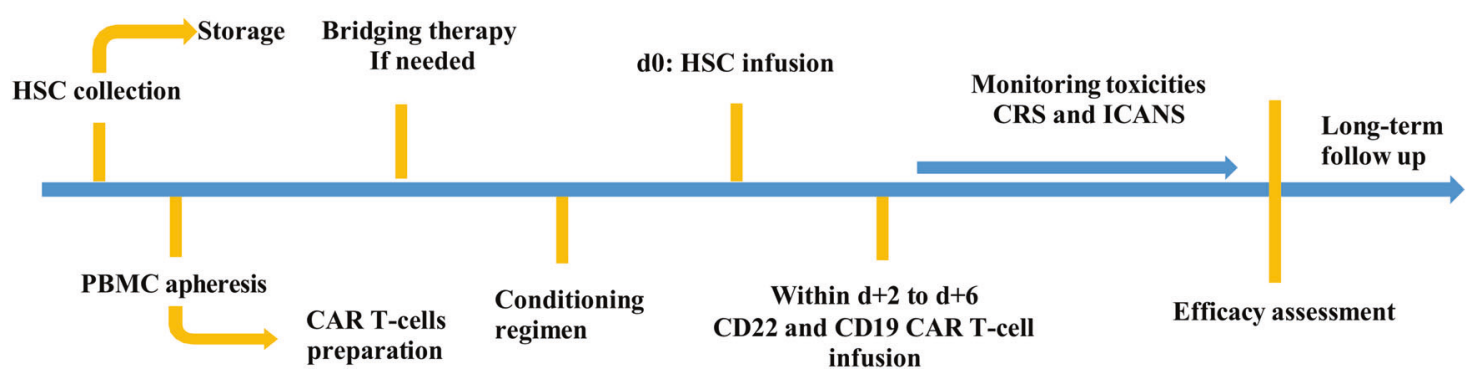

Fig. 1 Therapy procedure. All eligible CNSL patients underwent two separate apheresis, and received conditioning regimen before HSC infusion on d0, two separate CAR T-cell products (CD22 and CD19 CAR T-cells) were infused within the range of 2-6 days (d +2 to $d+6)$ after HSC infusion.

28,2021 , to observe the clinical efficacy and toxicity of sequential CD19/22 CAR T-cell immunotherapy following ASCT for CNSL. The trial was approved by the ethics committee of Tongji Hospital, Tongji Medical College, Huazhong University of Science and Technology. Informed consent was obtained by eligible patients and their families according to the Declaration of Helsinki.

\section{Therapy procedures}

All enrolled patients received two separate apheresis procedures before the conditioning regimen, including disease-sensitive chemotherapy combined with granulocyte colony-stimulating factor (G-CSF)-stimulated autologous hematopoietic stem cell (HSC) collection and peripheral blood mononuclear cell (PBMC) apheresis for CAR T-cell manufacturing. CAR Tcell manufacturing-related quality control and analysis were completed by Wuhan Bio-Raid Biotechnology Co., Ltd., as previously described [10]. Patients could receive bridging therapy to further reduce the tumor burden before receiving the conditioning regimen at the discretion of professional physicians.

The conditioning regimen mainly included a thiotepa-based protocol and the BEAM protocol. The BEAM protocol included carmustine $300 \mathrm{mg} /$ $\left(\mathrm{m}^{2} \cdot \mathrm{d}\right)$ for day -6 , etoposide $200 \mathrm{mg} /\left(\mathrm{m}^{2} \cdot \mathrm{d}\right)$ from days -5 to -2 , cytarabine $400 \mathrm{mg} /\left(\mathrm{m}^{2} \cdot \mathrm{d}\right)$ from days -5 to -2 , and melphalan $140 \mathrm{mg} /$ $\left(\mathrm{m}^{2} \cdot \mathrm{d}\right)$ for day -1 ; doxorubicin was given if needed. The thiotepa-based protocol included thiotepa $250 \mathrm{mg} /\left(\mathrm{m}^{2} \cdot \mathrm{d}\right)$ from days -9 to -7 , busulfan $3.2 \mathrm{mg} /(\mathrm{kg} \cdot \mathrm{d})$ from days -6 to -4 , and cyclophosphamide $60 \mathrm{mg} /(\mathrm{kg} \cdot \mathrm{d})$ from days -3 to -2 . Detailed dosages were adjusted at the physicians' discretion according to the fundamental status and tolerance of patients. Two separate CAR T-cell products (CD19 and CD22 CAR T-cells) were infused within the range of 2 to 6 days $(d+2$ to $d+6)$ after autologous HSC infusion (d0), and the infusion of CD22 CAR T-cell was usually one day prior to CD19 CAR T-cell considering the tolerance of patients. The therapy procedure was showed in Fig. 1.

\section{Efficacy and toxicity assessment}

Neutrophil engraftment was defined as the first of three consecutive days with an absolute neutrophil count $\geq 0.5^{*} 10^{9} / \mathrm{L}$. Response evaluation depended on the International PCNSL Collaborative Group Response Criteria and Lugano Response Criteria for B-Cell Lymphoma [2, 17]. CRS and ICANS were evaluated and graded according to the ASTCT Consensus Criteria $[18,19]$, and intervention therapies such as glucocorticoids or tocilizumab were given immediately according to the severity of CRS/ ICANS and patient tolerance [20, 21]. Progression-free survival (PFS) was defined as the time from ASCT to progression, death or the last follow-up point, and overall survival (OS) was presented as the time from ASCT to death or the last visit [22]. Multiparameter flow cytometry was used to detect the CD19/22 CAR T-cell percentage in peripheral blood (PB) and cerebrospinal fluid (CSF). The expansion of CAR T-cells in vivo was determined by droplet digital polymerase chain reaction (ddPCR). Fluorescence in situ hybridization (FISH) was performed to detect the amplification or translocation of $M Y C, B C L 2$, and $B C L 6$. Next-generation exome sequencing or HiSeq deep sequencing of 173 lymphoma-related genes was used for genetic mutation examination.

\section{Statistical analysis}

All statistical analyses were performed using SPSS 25.0 and GraphPad Prism 8.0. Continuous variables are reported as medians and ranges, and categorical variables are reported as frequencies and percentages.
The probability rates of PFS and OS were analyzed by the Kaplan-Meier method.

\section{RESULTS}

\section{Patient characteristics}

In total, 13 CNSL patients with a median age of 42 years (range: 23-65 years) were enrolled in our study from January 1, 2019, to February 28, 2021. These patients comprised six (46.2\%) males and seven $(53.8 \%)$ females. Of the 13 patients, including four PCNSL and nine SCNSL patients, 10 were detected by FISH, and abnormal genetic factors mainly involved $B C L 6 / M Y C$ rearrangement/amplification and TP53 deletion. BCL6 and MYC rearrangements simultaneously occurred in case 8 , which was diagnosed as "double hit" lymphoma. HiSeq deep sequencing was performed in nine patients, and with the exception of case 11, the remaining eight patients had positive pathogenic gene mutations, as shown in Table 1. Three of four patients with primary CNS DLBCL (cases 2, 6,13 ) failed to completely respond to first-line therapy, and the remaining patient (case 10) achieved CR through a combination of surgery and HD-MTX-based chemotherapy but relapsed after long-term follow-up; this patient later reached CR status again after treatment with tumor-sensitive chemotherapy before enrolling in our study. Among the nine SCNSL patients, two (cases 1 and 4) had CNS involvement at the initial diagnosis, three (cases 7, 9, and 12) systemic NHL patients were refractory to chemotherapy and experienced CNS involvement in the course of treatment, and the remaining four (cases 3,5,8, and 11) systemic NHL patients previous with CR status developed CNS recurrence. At the time of enrollment in our study, patients 7 and 12 had coexisting CNS lesions and active lymph nodes. The details of previous therapy for all patients are shown in Supplement 1. More basic information is shown in Table 1.

\section{Response and survival}

All patients received a conditioning regimen before HSC infusion; $38.5 \%(5 / 13)$ of sufferers used a thiotepa-based protocol, and $61.5 \%(8 / 13)$ chose the BEAM regimen. The median dosage of CD34 ${ }^{+}$cell infusion was $8.4(2.0-33.4) * 10^{6} / \mathrm{kg}$, the CD22 and CD19 CAR T-cells were separately infused, and the median cell number were $4.1(2.6-8.4) * 10^{6} / \mathrm{kg}$ and $4.3(2.0-9.2) * 10^{6} / \mathrm{kg}$, respectively. Enrolled patients achieved successful neutrophil engraftment with a median period of 13 days (range: 9-20 days) (Table 2).

Among the enrolled 13 patients, case 1 achieved CR status by receiving two separate CAR T-cell products (CD22 and CD19 CAR T-cells) "cocktail" treatment before enrollment, and case 10 also was MRI negative, which attributed to tumor-sensitive chemotherapy, both of the above patients received our protocol therapy for further consolidation, and maintained clinical efficacy without recurrence until the last visit. Nine of the remaining 11 patients responded to CD22/19 CAR T-cell immunotherapy following ASCT within 3 months, including six who achieved $C R$ and three who achieved partial remission (PR). The overall response rate (ORR) 

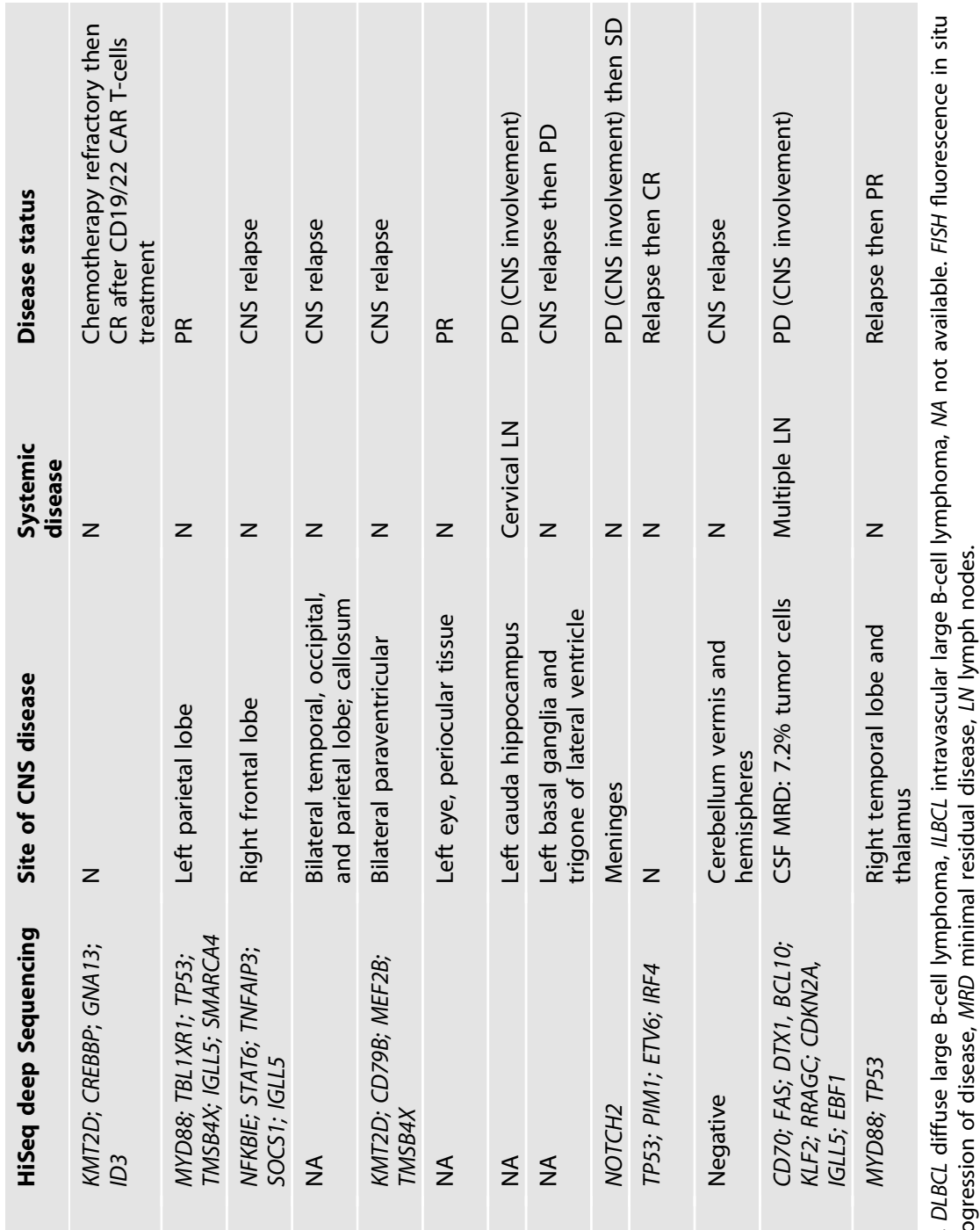

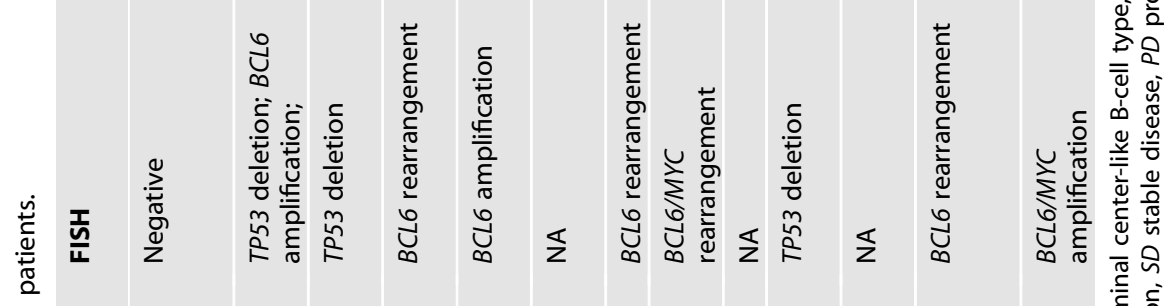

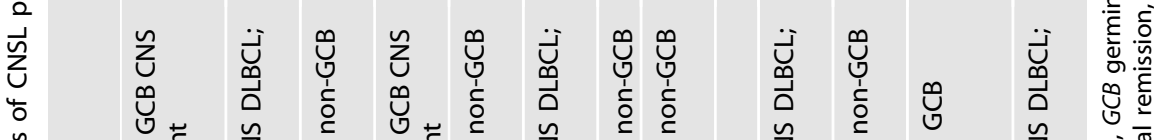

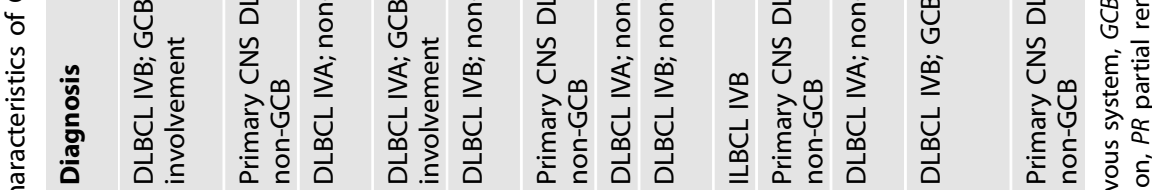

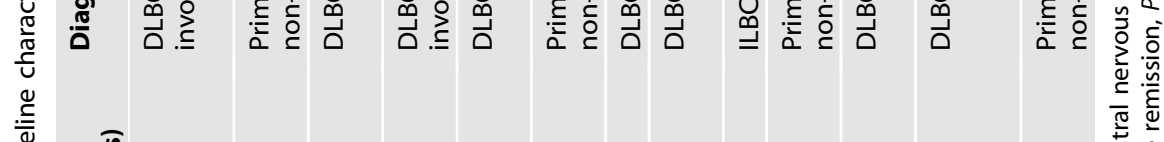

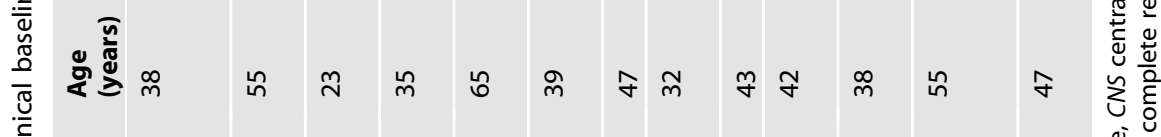

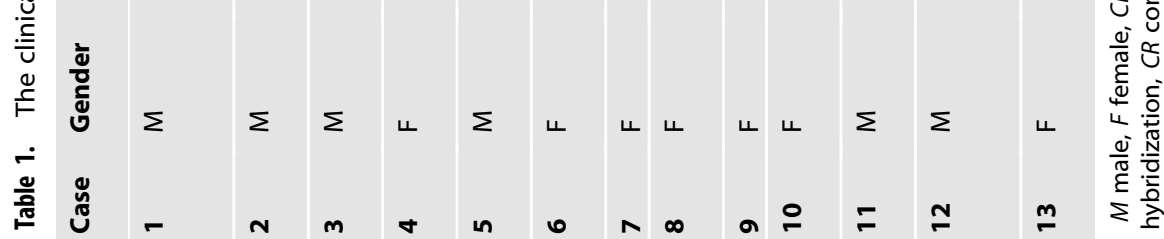




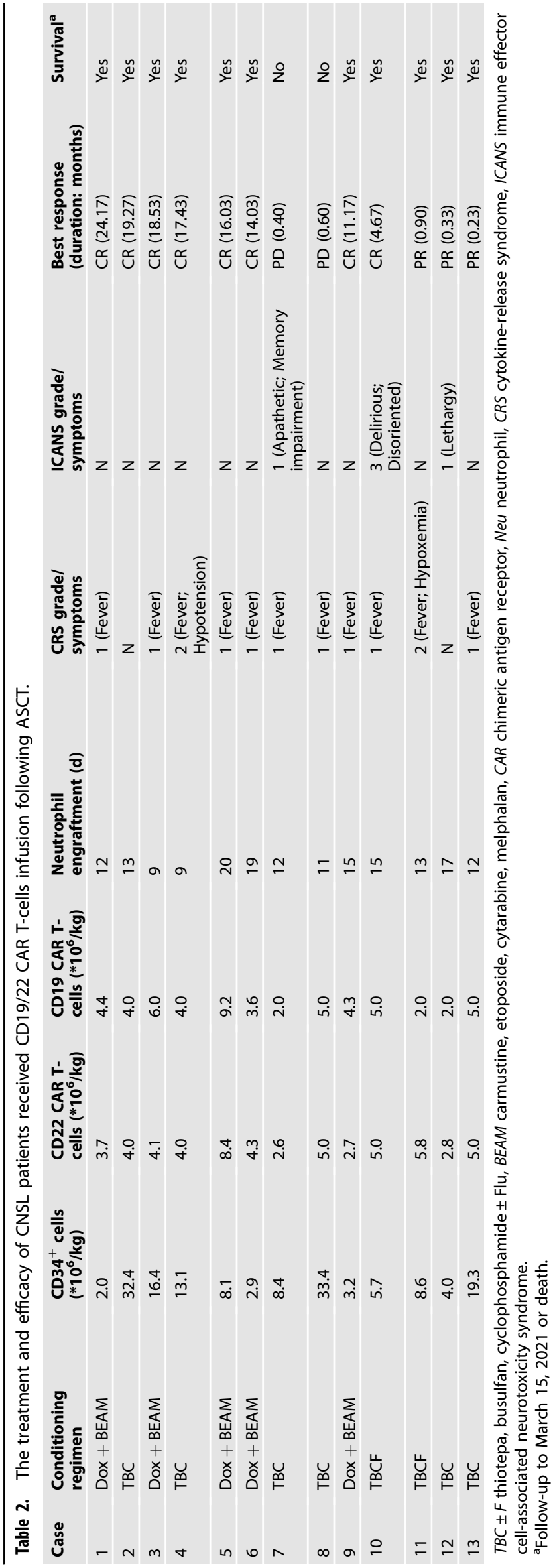

and complete remission rate (CRR) were $81.81 \%$ and $54.55 \%$, respectively. Patient 11 developed disease progression after achieving PR status in the first efficacy assessment. The median durable time for the nine responsive patients was 14.03 months (range: $0.23-19.27$ months); $18.18 \%(2 / 11)$ of patients (cases 7 and 8 ) did not respond to the current treatment protocol and maintained progressive disease (PD) status (Fig. 2A). MRI images for three representative patients before therapy and at the time of best response are shown in Fig. 2B.

As of March 15, 2021, the median follow-up time of all enrolled patients was 14.20 months (range: 1.37-24.17 months), and only two systemic NHL patients with CNS involvement/relapse (cases 7 and 8 ) refused further treatment due to multiple virus infection and/or rapid disease progression. Both died shortly after leaving hospital, with a median survival time of 2.33 months (range: 1.5-3.17 months). The median PFS and OS after ASCT and CAR Tcell infusion were undefined, and the estimated 1-year PFS and OS rates were $74.59 \%$ (95\% Cl: $39.76-91.10 \%)$ and $82.50 \%(95 \% \mathrm{Cl}$ : 46.10-95.33\%), respectively (Fig. 2C).

\section{Side effects}

After CAR T-cell infusion, 11 (84.62\%) patients (except cases 2 and 12) had low-grade CRS. Nine had fever over $38^{\circ} \mathrm{C}$ and were assessed as having grade 1 CRS, and two exhibited grade 2 CRS and presented with fever and hypotension or hypoxemia; no patient suffered grades 3-4 CRS. In addition, case 7 presented with apathetic and mild memory impairment (ICE score: 8 points) and case 12 with lethargy (ICE score: 9 points) both were assessed as grade 1 ICANS. Patient 10, who appeared delirious and disoriented (ICE score: 1 point), was diagnosed with grade 3 ICANS. The cumulative incidence of ICANS was $27.27 \%$. Grade 1 CRS cases were reversed after timely administration of broad-spectrum antibiotics, while patients with ICANS or grade 2 CRS were recovered by simultaneously combing with intravenous methylprednisolone injection, no patient had residual neurological impairment. Other side effects included viremia (4/13) and upper respiratory infection (1/13); one (case 7) patient refused further therapy because of multiple virus infection (CMV, JCV, BKV, TTV) and disease progression, and others were supported by effective anti-infection treatment. As shown in Fig. 3A, the developed ferritin index varied, increasing from the baseline level except for cases 9 and 13, as did the IL-6 level, excluding cases 1 and 12. Ferritin and IL-6 reached the peak levels (ferritin: $2235 \mu \mathrm{g} / \mathrm{L} ; \mathrm{IL}-6: 194 \mathrm{pg} / \mathrm{mL}$ ) on the median 8th and 7th day after the first CAR T-cell infusion, respectively. In addition, increases in serum ferritin and IL- 6 levels were often accompanied by CRS and ICANS, and patients who experienced grade 2 CRS or grade 3 ICANS had peak serum ferritin and IL-6 levels greater than $3000 \mu \mathrm{g} / \mathrm{L}$ and $300 \mathrm{pg} / \mathrm{mL}$, respectively.

\section{CAR T-cell kinetics}

Except for patient 10, the remaining 12 patients underwent ddPCR testing for absolute quantification of CAR gene expression to better understand the expansion of CAR T-cells in vivo. As shown in Fig. 3B, the median peak numbers of CD19 and CD22 CAR Tcells lentivirus copies in vivo were 911 (range: 33-6900) and 3346

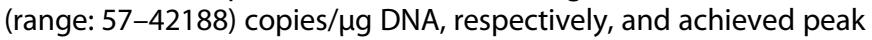
levels with median times of 1.36 (range: $0.72-1.86$ ) and 1.46 (range: $0.43-3.00$ ) weeks after CAR T-cell infusion, respectively. Interestingly, the lentivirus copies of CD19 and CD22 CAR T-cells in all patients except patient 8 (who suffered early death without explicit data) were unabiding, only with median durable times of $8.43(1.29-20.72)$ and 8.57 (2.00-45.43) weeks, respectively. The CAR T-cells percentage in CD3 + cells was measured by flow cytometry; only case 2 received regular detection monthly for the first 3 months after CAR T-cells infusion, followed by evaluations at $\sim 6$ and 12 months, and the peak percentages of CAR T-cells in CD3 + cells in the CSF and PB were $46.38 \%$ and $17.58 \%$, respectively. 
A

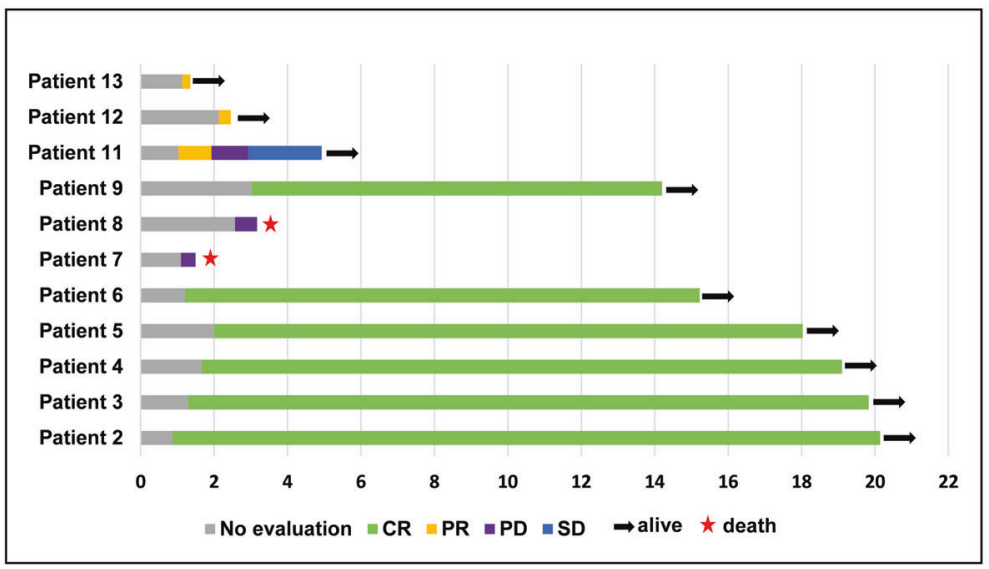

B

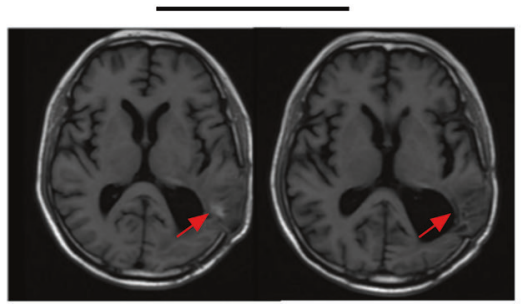

Pt 4

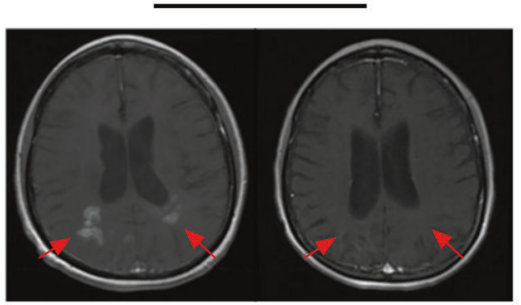

C

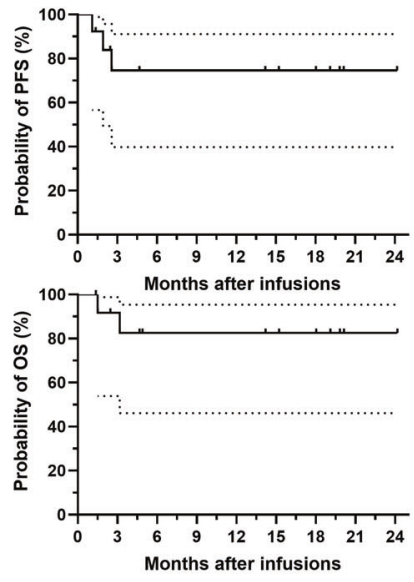

Pt 9

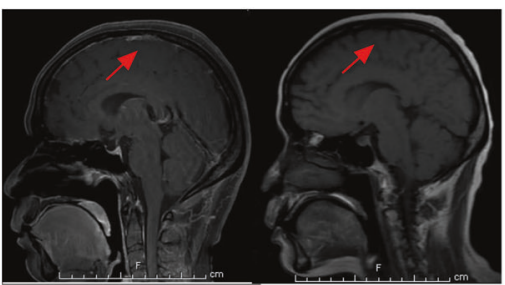

Fig. 2 Descriptions of the clinical results. A The clinical outcomes (excluded patient 1 and 10), the last visit day was March $15,2020$. B Representative MRI imaging before (left) and after (right) therapy. C The probability of PFS and OS. The median PFS and OS of patients were undefined, the 1-year estimated PFS and OS rate were $74.59 \%$ (95\% Cl: $39.76-91.10 \%)$ and $82.50 \%$ (95\% Cl: $46.10-95.33 \%)$, respectively.

A

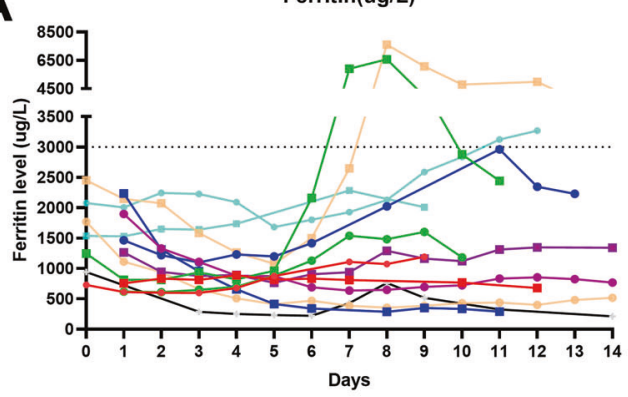

IL-6(pg/ml)

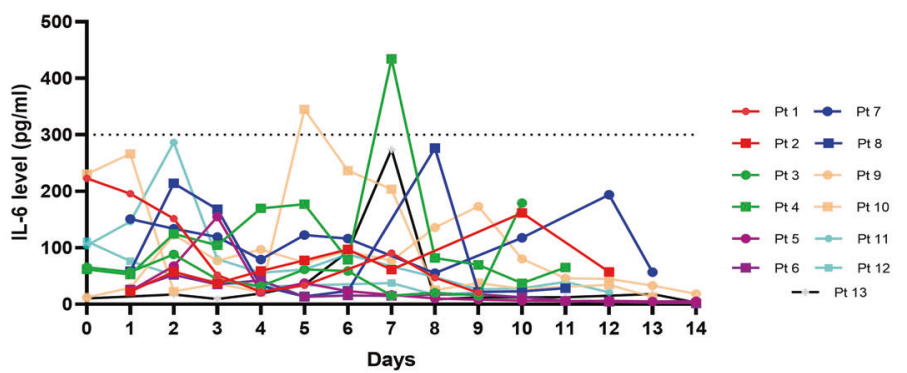

B
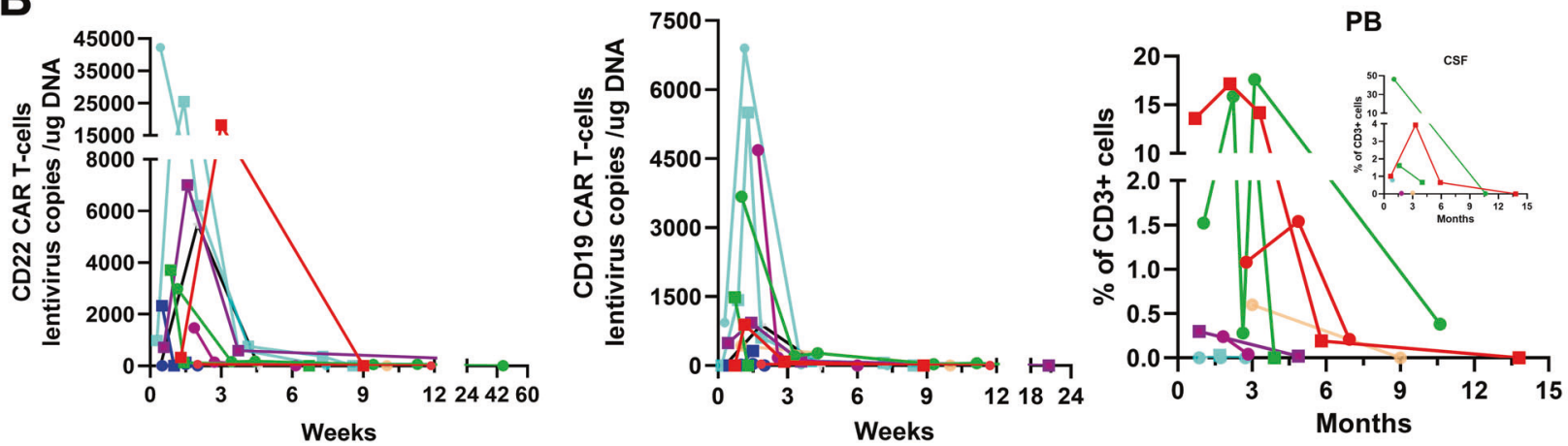

Fig. 3 The variation of inflammatory markers and CAR T-cell kinetics. A The serum ferritin and IL-6 level of patients. Increases in serum ferritin and IL-6 level were often accompanied by CRS and ICANS. B Lentivirus copies in vivo and percentage of CAR T-cells in CD3 + cells. The median peak number of CD19 and CD22 CAR T-cells lentivirus copies were 911 and 3346 copies/ $\mu$ g DNA, and the peak percentage of CAR T-cells in CD3 + cells in CSF and PB were $46.38 \%$ and $17.58 \%$, respectively. 


\section{DISCUSSION}

Recently, the "living" drug of CAR T-cell infusion following ASCT has been demonstrated to be relatively effective and safe for $r / r$ multiple myeloma and B-cell NHL [22-24]. Several studies have also reported the clinical efficacy of CNSL receiving separate CAR T-cell immunotherapy, but limited data on ASCT sequential CAR Tcell infusion have been reported [15, 25, 26]. Herein, we reported the clinical efficacy and toxicities of 13 CNSL patients (four PCNSL and nine SCNSL) received sequential CD19/22 CAR T-cell immunotherapy following ASCT, no patient suffered grades 3-4 CRS, and only one patient experienced severe immune ICANS. The ORR and CRR were $81.81 \%$ and $54.55 \%$, and the estimated 1 -year PFS and OS rates were $74.59 \%$ and $82.50 \%$, respectively. Our preliminary results showed that CNSL patients who received sequential CD19/22 CAR T-cell immunotherapy following ASCT achieved long-term remission without serious toxicities.

In our present study, two patients with CR status were voluntarily enrolled in our trial for further consolidation considering the aggressive progression of CNSL, and both achieved long-term disease/relapse-free survival, indicating that ASCT sequential CAR Tcell infusion can have a long-term consolidation effect for $r / r$ highrisk CNSL patients. In addition, nine of the remaining 11 patients responded within 3 months after treatment, and the duration of recurrence-free $C R$ was more than a year in five of six patients. This is inconsistent with previous reports that some patients with CNSL who received CAR T-cell infusion or ASCT can obtain an objective clinical response but not an enduring response $[6,16]$. The main reasons for this difference are as follows. First, a conditioning regimen and ASCT can minimize the tumor burden and disease activity and deeply deplete lymphocytes that inhibit CAR T-cell function [22]. Second, the ability of HSC to improve the complex and unique tumor immunosuppressive microenvironment of the CNS may be beneficial to reduce the possibility of relapse [27]. Last, the target killing effect of CAR T-cells to purify the graft can effectively avoid the recurrence caused by product contamination [28, 29].

However, several (2/13) patients did not respond to our treatment and died of rapid disease progression, with a median survival time of 2.33 months; however, no deaths were attributed to CAR T-cell products. The two unresponsive patients were both in PD status before our enrollment, and the lack of response may be associated with rapid growth of tumor cells counteracting the effects of HSCs and CD19/22 CAR T-cells. A significant proportion of aggressive disease patients lose the opportunity for CAR T-cell infusion due to rapid progression or clinical deterioration, so other preferential therapies should be given to maximize killing tumor cell populations to improve the therapeutic effect before ASCT sequential CAR T-cell immunotherapy, especially for patients with PD or stable disease status accompanied by high tumor burden [22]. Patient 8, with highrisk double hit (BCL6 and MYC rearrangement) disease, belongs to a population with historically poor prognosis, which may also result in a nonresponsive outcome [30,31].

All patients reached peak levels of lentiviral copies of CD19/22 CAR T-cells within 2 weeks, which was consistent with previous reports on hematological malignancies receiving CAR T-cell therapy $[32,33]$; however, another attractive phenomenon was the undetectable lentiviral copies of CD19/22 CAR T-cells in vivo within a median time of $<3$ months after infusion. Even in patients with a durable response, the sustainable effects did not appear to require prolonged expansion and persistence of functional CAR Tcells. The mechanism of this phenomenon still needs to be investigated. We speculate that it may be related to the lack of effective antigen stimulation in durable remission patients based on the past conclusion that low CD22 expression impaired in vivo CD22 CAR T-cell persistence [34]. In addition, a small portion of effector CAR T-cells may have been induced and transformed into durable memory CAR T-cells to promptly exert the antitumor effect upon recurrence [35], but the level in the blood is lower than the detectable threshold of present quantification technology. More sensitive and accurate detection techniques for the quantification of transgenic CAR T-cells may contribute to increasing the number of positive results $[36,37]$.

CRS and ICANS, as the most common CAR T-cell-associated side effects, should be closely monitored, and previous studies on CAR T-cell immunotherapy have excluded CNSL patients due to concerns about potentially fatal ICANS [14, 25]. In our present study, most patients developed fever $\left(\geq 38^{\circ} \mathrm{C}\right)$ after CAR T-cell infusion and were assessed as having grade $1 \mathrm{CRS}$. No patient experienced grades 3-4 CRS, and only one patient suffered grade 3 severe ICANS. For all patients, these symptoms were reversible by timely therapy, and no patient died of severe side toxicities. Previously, a multicenter, phase 2 trial [38] conducted by Neelapu et al. in 111 DLBCL patients who received CD19 CAR T-cell therapy indicated that grades 3 or higher CRS and ICANS occurred in 13\% and $28 \%$ of recipients, respectively. No obvious difference or elevation was observed in our current research, occasionally, the results were even better than those of separate CAR T-cell therapy, which was also consistent with other earlier related reports, including those related to ASCT sequential CAR T-cell infusion for other hematological malignancies [10, 24, 25, 38, 39]. The acceptable CRS and ICANS rates may be attributed to the use of an enhanced conditioning regimen and ASCT before CAR T-cell infusion to minimize the tumor burden and myeloid cells, which have been confirmed to be related to the occurrence and severity of CRS and ICANS [39-41]; moreover, myeloid cell-derived cytokines (IL-1, IL-6, GM-CSF, etc.) were the major sources of CRS after CAR T-cell immunotherapy [42-44]. Of course, the manageable side effects can also be attributed to the development of supportive therapy and CAR T-cell manufacturing-related technology. Taken together, our research further indicated that CNSL no longer has to be considered a contraindication to CAR T-cell immunotherapy and that ASCT sequential CD19/22 CAR T-cell therapy for CNSL patients proved to have low toxicity.

Meanwhile, we detected the daily levels of IL- 6 and ferritin during hospitalization and attempted to analyze the relationship between the levels of serum cytokines and inflammatory markers and the occurrence of CRS and ICANS. Consistent with earlier studies, we observed that elevated serum ferritin and IL-6 levels were often accompanied by CRS and ICANS; moreover, the degree was positively related to the ferritin and IL-6 levels [38, 45-47]. The relationships among CRS, ICANS and other cytokines (such as IL-10 and interferon$\mathrm{\gamma})$ and indicators (such as angiopoietin and von Willebrand factor) need to be monitored and analyzed in future studies.

In general, our preliminary experience demonstrated that sequential CD19/22 CAR T-cell immunotherapy following ASCT acted as a novel therapy for CNSL and appears to have encouraging long-term efficacy with relatively manageable side effects. However, there are some limitations in our present study. The number of cases for analysis was small, and the follow-up time for some patients was relatively short. It is necessary to proceed with multicenter prospective research to further clarify the clinical efficacy and safety of CD19/22 CAR T-cell immunotherapy following ASCT for CNSL. Meanwhile, a clinical trial comparing ASCT and CAR T-cell therapy with ASCT or CAR T-cells alone in treating CNSL patients should be conducted.

\section{REFERENCES}

1. Chukwueke UN, Nayak L. Central nervous system lymphoma. Hematol Oncol Clin North Am. 2019;33:597-611.

2. Han $\mathrm{CH}$, Batchelor $\Pi$. Diagnosis and management of primary central nervous system lymphoma. Cancer. 2017;123:4314-24.

3. Holdhoff M, Mrugala MM, Grommes C, Kaley TJ, Swinnen LJ, Perez-Heydrich C, et al. Challenges in the treatment of newly diagnosed and recurrent primary central nervous system lymphoma. J Natl Compr Canc Netw. 2020;18:1571-8.

4. Ferreri $A J$, Illerhaus $G$. The role of autologous stem cell transplantation in primary central nervous system lymphoma. Blood. 2016;127:1642-9. 
5. El-Galaly TC, Cheah CY, Bendtsen MD, Nowakowski GS, Kansara R, Savage KJ, et al. Treatment strategies, outcomes and prognostic factors in 291 patients with secondary CNS involvement by diffuse large B-cell lymphoma. Eur J Cancer. 2018;93:57-68.

6. Houillier $C$, Soussain $C$, Ghesquières $H$, Soubeyran $P$, Chinot $O$, Taillandier $L$, et al. Management and outcome of primary CNS lymphoma in the modern era: an LOC network study. Neurology. 2020;94:e1027-e1039.

7. Nevel KS, Pentsova E, Daras M. Clinical presentation, treatment, and outcomes of patients with central nervous system involvement in extranodal natural killer/Tcell lymphoma. Leuk Lymphoma. 2019;60:1677-84.

8. Ying Z, Huang XF, Xiang X, Liu Y, Kang X, Song Y, et al. A safe and potent antiCD19 CAR T cell therapy. Nat Med. 2019;25:947-53.

9. Cooper ML, DiPersio JF. Chimeric antigen receptor T cells (CAR-T) for the treatment of T-cell malignancies. Best Pract Res Clin Haematol. 2019;32:101097.

10. Wang N, Hu X, Cao W, Li C, Xiao Y, Cao Y, et al. Efficacy and safety of CAR19/22 Tcell cocktail therapy in patients with refractory/relapsed B-cell malignancies. Blood. 2020;135:17-27.

11. Martyniszyn A, Krahl AC, André MC, Hombach AA, Abken H. CD20-CD19 bispecific CAR T cells for the treatment of B-cell malignancies. Hum Gene Ther. 2017;28:1147-57.

12. Song MK, Park BB, Uhm JE. Resistance mechanisms to CAR T-cell therapy and overcoming strategy in B-cell hematologic malignancies. Int $\mathrm{J}$ Mol Sci. 2019;20:5010.

13. Cheng J, Zhao L, Zhang Y, Qin Y, Guan Y, Zhang T, et al. Understanding the mechanisms of resistance to CAR T-cell therapy in malignancies. Front Oncol. 2019;9:1237.

14. Karschnia P, Jordan JT, Forst DA, Arrillaga-Romany IC, Batchelor TT, Baehring JM, et al. Clinical presentation, management, and biomarkers of neurotoxicity after adoptive immunotherapy with CAR T cells. Blood. 2019;133:2212-21.

15. Tu S, Zhou X, Guo Z, Huang R, Yue C, He Y, et al. CD19 and CD70 dual-target chimeric antigen receptor T-cell therapy for the treatment of relapsed and refractory primary central nervous system diffuse Large B-cell lymphoma. Front Oncol. 2019:9:1350.

16. Li T, Tan J, Chen L, Kuang D, Mao X, Lou Y, et al. CAR T-cell therapy is effective but not long-lasting in B-cell lymphoma of the brain. Front Oncol. 2020;10:1306.

17. Zelenetz AD, Gordon LI, Abramson JS, Advani RH, Bartlett NL, Caimi PF, et al. NCCN guidelines insights: B-cell lymphomas, version 3.2019. J Natl Compr Canc Netw. 2019;17:650-61.

18. Freyer CW, Porter DL. Cytokine release syndrome and neurotoxicity following CAR T-cell therapy for hematologic malignancies. J Allergy Clin Immunol. 2020;146:940-8.

19. Lee DW, Santomasso BD, Locke FL, Ghobadi A, Turtle CJ, Brudno JN, et al. ASTCT consensus grading for cytokine release syndrome and neurologic toxicity associated with immune effector cells. Biol Blood Marrow Transplant. 2019;25:625-38.

20. Brudno JN, Kochenderfer JN. Toxicities of chimeric antigen receptor T cells: recognition and management. Blood. 2016;127:3321-30.

21. Frey N. Cytokine release syndrome: Who is at risk and how to treat. Best Pract Res Clin Haematol. 2017;30:336-40.

22. Wang T, Gao L, Wang Y, Zhu W, Xu L, Wang Y, et al. Hematopoietic stem cell transplantation and chimeric antigen receptor $\mathrm{T}$ cell for relapsed or refractory diffuse large B-cell lymphoma. Immunotherapy. 2020;12:997-1006.

23. Garfall AL, Stadtmauer EA, Hwang WT, Lacey SF, Melenhorst JJ, Krevvata M, et al. Anti-CD19 CAR T cells with high-dose melphalan and autologous stem cell transplantation for refractory multiple myeloma. JCI Insight. 2018;3: e120505

24. Sauter CS, Senechal B, Rivière I, Ni A, Bernal Y, Wang X, et al. CD19 CAR T cells following autologous transplantation in poor-risk relapsed and refractory B-cell non-Hodgkin lymphoma. Blood. 2019;134:626-35.

25. Frigault MJ, Dietrich J, Martinez-Lage $M$, Leick $M$, Choi BD, DeFilipp $Z$, et al. Tisagenlecleucel CAR T-cell therapy in secondary CNS lymphoma. Blood. 2019;134:860-6.

26. Abramson JS, McGree B, Noyes S, Plummer S, Wong C, Chen YB, et al. Anti-CD19 CAR T cells in CNS diffuse large-B-cell lymphoma. N Engl J Med. 2017;377:783-4.

27. Wrzesinski C, Paulos CM, Gattinoni L, Palmer DC, Kaiser A, Yu Z, et al. Hematopoietic stem cells promote the expansion and function of adoptively transferred antitumor CD8 T cells. J Clin Invest 2007;117:492-501.

28. Fouillard L, Laporte JP, Labopin M, Lesage S, Isnard F, Douay L, et al. Autologous stem-cell transplantation for non-Hodgkin's lymphomas: the role of graft purging and radiotherapy posttransplantation-results of a retrospective analysis on 120 patients autografted in a single institution. J Clin Oncol. 1998;16:2803-16.

29. Yahng SA, Yoon JH, Shin SH, Lee SE, Cho BS, Eom KS, et al. Influence of ex vivo purging with CliniMACS CD34(+) selection on outcome after autologous stem cell transplantation in non-Hodgkin lymphoma. Br J Haematol. 2014;164:555-64.

30. Riedell PA, Smith SM. Double hit and double expressors in lymphoma: definition and treatment. Cancer. 2018;124:4622-32.
31. Rosenthal A, Younes A. High grade B-cell lymphoma with rearrangements of MYC and BCL2 and/or BCL6: double hit and triple hit lymphomas and double expressing lymphoma. Blood Rev. 2017;31:37-42.

32. Maude SL, Laetsch TW, Buechner J, Rives S, Boyer M, Bittencourt H, et al. Tisagenlecleucel in children and young adults with B-cell lymphoblastic leukemia. N Engl J Med. 2018;378:439-48.

33. Schuster SJ, Bishop MR, Tam CS, Waller EK, Borchmann P, McGuirk JP, et al. Tisagenlecleucel in adult relapsed or refractory diffuse large B-cell lymphoma. $\mathrm{N}$ Engl J Med. 2019;380:45-56.

34. Ramakrishna S, Highfill SL, Walsh Z, Nguyen SM, Lei H, Shern JF, et al. Modulation of target antigen density improves CAR T-cell functionality and persistence. Clin Cancer Res. 2019;25:5329-41.

35. Kalos M, Levine BL, Porter DL, Katz S, Grupp SA, Bagg A, et al. T cells with chimeric antigen receptors have potent antitumor effects and can establish memory in patients with advanced leukemia. Sci Transl Med. 2011;3:95ra73.

36. Baras A, Bocket L, Beauvais D, Hallaert C, Varlet $P$, Yakoub-Agha I, et al. A real-time quantitative PCR targeting the viral vector for the monitoring of patients treated with axicabtagene ciloleucel. J Mol Diagn. 2021;23:447-54.

37. Schubert ML, Kunz A, Schmitt A, Neuber B, Wang L, Hückelhoven-Krauss A, et al. Assessment of CAR T cell frequencies in axicabtagene ciloleucel and tisagenlecleucel patients using duplex quantitative PCR. Cancers (Basel). 2020;12:2820.

38. Neelapu SS, Locke FL, Bartlett NL, Lekakis L, Miklos DB, Jacobson CA, et al Axicabtagene ciloleucel CAR T-cell therapy in refractory large B-Cell lymphoma. $\mathrm{N}$ Engl J Med. 2017;377:2531-44.

39. Curran KJ, Margossian SP, Kernan NA, Silverman LB, Williams DA, Shukla N, et al. Toxicity and response after CD19-specific CAR T-cell therapy in pediatric/young adult relapsed/refractory B-ALL. Blood. 2019;134:2361-8.

40. Park JH, Rivière I, Gonen $M$, Wang $X$, Sénéchal $B$, Curran $K J$, et al. Long-term follow-up of CD19 CAR therapy in acute lymphoblastic leukemia. N Engl J Med. 2018;378:449-59.

41. Wei J, Liu Y, Wang C, Zhang Y, Tong C, Dai G, et al. The model of cytokine release syndrome in CAR T-cell treatment for B-cell non-Hodgkin lymphoma. Signal Transduct Target Ther. 2020;5:134.

42. Giavridis T, van der Stegen S, Eyquem J, Hamieh M, Piersigilli A, Sadelain M. CAR T cell-induced cytokine release syndrome is mediated by macrophages and abated by IL-1 blockade. Nat Med. 2018;24:731-8.

43. Norelli M, Camisa B, Barbiera G, Falcone L, Purevdorj A, Genua M, et al. Monocytederived IL-1 and IL- 6 are differentially required for cytokine-release syndrome and neurotoxicity due to CAR T cells. Nat Med. 2018;24:739-48.

44. Sachdeva M, Duchateau P, Depil S, Poirot L, Valton J. Granulocyte-macrophage colony-stimulating factor inactivation in CAR T-cells prevents monocytedependent release of key cytokine release syndrome mediators. J Biol Chem 2019;294:5430-7

45. Wang Z, Han W. Biomarkers of cytokine release syndrome and neurotoxicity related to CAR-T cell therapy. Biomark Res. 2018;6:4.

46. Teachey DT, Lacey SF, Shaw PA, Melenhorst JJ, Maude SL, Frey N, et al. Identification of predictive biomarkers for cytokine release syndrome after chimeric antigen receptor T-cell therapy for acute lymphoblastic leukemia. Cancer Discov. 2016;6:664-79.

47. Brudno JN, Kochenderfer JN. Recent advances in CAR T-cell toxicity: mechanisms, manifestations and management. Blood Rev. 2019;34:45-55.

\section{ACKNOWLEDGEMENTS}

The authors sincerely thank all patients and their families, as well as Wuhan Bio-Raid Biotechnology Co., Ltd. and our colleagues. This work was supported by the National Natural Science Foundation of China (No. 81873444 \& 82070213), Key R \& D project of Hubei science and Technology Department (No. 202031) and Project of Hubei Provincial Health Commission (WJ2019M126).

\section{AUTHOR CONTRIBUTIONS}

JW collected, analyzed the data and wrote the paper; $Y X$ helped to review and approve the final version for submission; JZ conceived and designed the study; all authors executed this clinical trial.

\section{COMPETING INTERESTS}

The authors declare no competing interests.

\section{ETHICS}

The trial was approved by the ethics committee of Tongji Hospital, Tongji Medical College, Huazhong University of Science and Technology. 


\section{INFORMED CONSENT}

Informed consent was obtained by eligible patients and their families according to the Declaration of Helsinki.

\section{ADDITIONAL INFORMATION}

Supplementary information The online version contains supplementary material available at https://doi.org/10.1038/s41408-021-00523-2.

Correspondence and requests for materials should be addressed to J.Z. or Y.X.

Reprints and permission information is available at http://www.nature.com/reprints

Publisher's note Springer Nature remains neutral with regard to jurisdictional claims in published maps and institutional affiliations.
Open Access This article is licensed under a Creative Commons Attribution 4.0 International License, which permits use, sharing, adaptation, distribution and reproduction in any medium or format, as long as you give appropriate credit to the original author(s) and the source, provide a link to the Creative Commons license, and indicate if changes were made. The images or other third party material in this article are included in the article's Creative Commons license, unless indicated otherwise in a credit line to the material. If material is not included in the article's Creative Commons license and your intended use is not permitted by statutory regulation or exceeds the permitted use, you will need to obtain permission directly from the copyright holder. To view a copy of this license, visit http://creativecommons. org/licenses/by/4.0/.

(c) The Author(s) 2021 